\title{
Margarita Burn: Recognition and Treatment of Phytophotodermatitis
}

\author{
Ganesh Maniam, MBA, Katelyn MacLeay Light, MD, and Joanna Wilson, DO
}

Phytophotodermatitis is a cutaneous reaction caused by direct contact with phototoxic agents and subsequent sunlight exposure. Furocoumarins and psoralens are 2 phototoxic agents that can cause this reaction, and these organic chemical compounds are found in many plant species consumed by humans. Following contact exposure to such foods and ultraviolet radiation exposure via direct sunlight, phytophotodermatitis can occur. Due to the etiology of these rashes relating closely to the outdoor consumption of margaritas, the rash may be known by patients as "margarita burn." There is a classically described sequence of rash progression: erythematous macules or patches, which later become vesicles and seem similar to second-degree burns, followed by an asymptomatic hyperpigmentation. This case presents a 26-year-old female diagnosed with phytophotodermatitis following use of citrus fruits for margaritas while outdoors in direct sunlight. The diagnosis of phytophotodermatitis is often made clinically but can be complicated due to its similarity in appearance to many other common cutaneous reactions. In this patient, the differential diagnosis included solar erythema, contact dermatitis (type IV hypersensitivity reaction), polymorphic light eruption, or drug-related photosensitivity. Careful history taking is essential in not only narrowing down the differential diagnosis but also in avoiding unnecessary tests or ineffective treatments. (J Am Board Fam Med 2021;34:398-401.)

Keywords: Citrus, Dermatology, Differential Diagnoses, Family Medicine, Furocoumarins, Medical History Taking, Phototoxic Dermatitis, Phytophotodermatitis, Type IV Hypersensitivity, Ultraviolet Rays

\section{Introduction}

Phytophotodermatitis is a cutaneous reaction caused by direct contact with phototoxic agents and subsequent sunlight exposure. ${ }^{1}$ Furocoumarins and psoralens are 2 such phototoxic agents that can cause this reaction, ${ }^{1}$ and these organic chemical compounds are found in many plant species consumed by humans. ${ }^{2}$ In certain species of edible plants, specifically the Rutaceae and Umbelliferae families, the synthesis of furocoumarins from psoralen precursors is necessary for predator defense or as a stress response. ${ }^{2}$ However,

This article was externally peer reviewed.

Submitted 23 July 2020; revised 28 September 2020; accepted 29 September 2020.

From the Texas Tech University Health Sciences Center, Amarillo (GM); Department of Family Medicine at Texas A\&M College of Medicine, Bryan/College Station (KML); Department of Internal Medicine at Texas Tech University Health Sciences Center, Amarillo (JW).

Funding: There are no funding sources to report.

Conflict of interest: None declared.

Corresponding author: Ganesh Maniam, MBA, 5509 SW 9th Ave., Apt. 315, Amarillo, TX 79106 (E-mail: Ganesh. Maniam@ttuhsc.edu). the consumption of various fruits and herbs from these families may lead to cutaneous contact with these phototoxic agents. ${ }^{2}$ Rutaceous plants with these agents include oranges, lemons, limes, and grapefruit; umbelliferous plants with these agents include carrots, celery, parsley, and parsnips. ${ }^{2}$ Following contact exposure to such foods and ultraviolet radiation exposure via direct sunlight, phytophotodermatitis can occur. ${ }^{3}$

Due to the etiology of these rashes relating closely to the outdoor consumption of margaritas, the rash may be known by patients as "margarita burn." The classically described clinical triad in these patients is a sequence of rash progression: first as erythematous macules or patches, which later become vesicles similar to second-degree burns, before finally becoming an asymptomatic hyperpigmentation. ${ }^{3}$ However, the wide variety of presentations may cause a difficult diagnosis due to confusion with other similar skin conditions. ${ }^{4}$ This case presents a 26 -year-old female diagnosed with phytophotodermatitis following use of citrus fruits for margaritas while outdoors in direct sunlight, and a discussion of this case as well as its 
Figure 1. Erythematous macules on day 1.

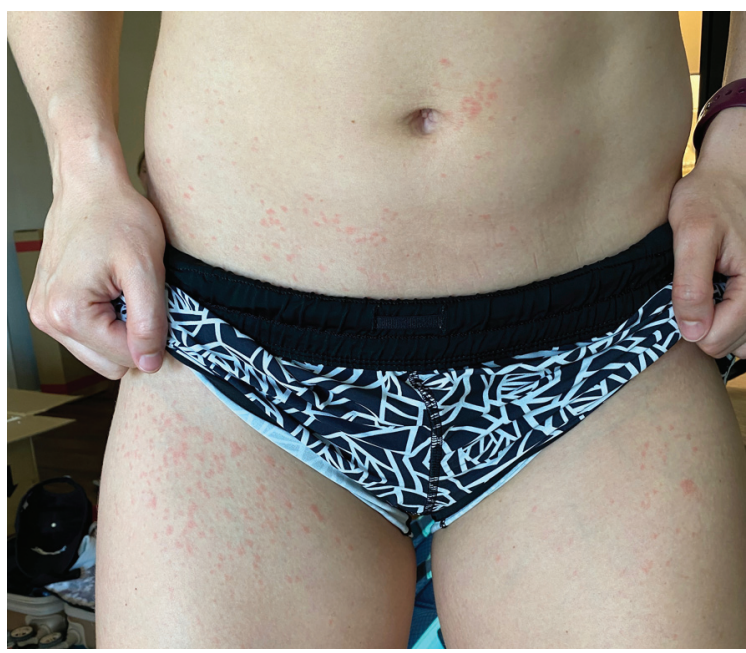

atypical features may assist in the recognition and treatment of similar cases.

\section{Case Description}

A 26-year-old female complains of a faint, but sharply demarcated, macular erythematous rash localized to the lower abdomen and upper thighs approximately 7 hours after slicing and squeezing 2 dozen limes to make margaritas for a pool party (Figure 1). The patient reports that she first felt a burning sensation in the area while getting into the pool, before the rash appeared later that day. She denies any history of a similar rash, denies any contact with any nearby plants, denies any new soaps or sunscreens, and denies any new clothing or swimwear being worn that day. She had used the same pool the previous weekend and denies any new cleaning agent being used in that pool. She denies any other symptoms throughout the afternoon. She was the only attendee at the pool party who developed this rash.

By the next morning, day 1, the rash had spread farther up the abdomen and around the thighs and had become more erythematous (pink) but was not tender or pruritic and remained sharply demarcated. By day 2, the rash became more maculopapular in appearance, and the patient described a "burning" sensation to the rash. On day 3, the patient reported an increase in the intensity of this burning sensation, and the rash became more widespread, tender, and erythematous (red); tense vesicles also began to form (Figure 2). The patient
Figure 2. Tense vesicles on day 3 .

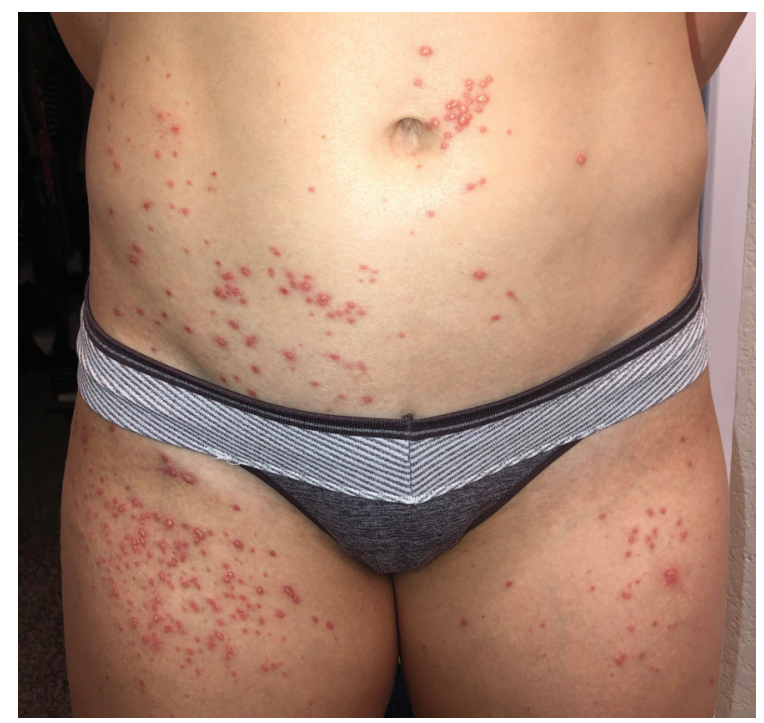

presented to her primary care physician and was prescribed oral prednisone (10 mg, BID) for 7 days as well as once daily application of topical steroid cream (hydrocortisone 1\%) for 7 days for unspecified dermatitis. By day 4, despite patient compliance with prescribed medications, these erythematous tense vesicles continue to increase in size as well as distribution-though remained demarcated. The patient also reported increased intensity of burning sensation and pain.

By the end of day 4, the vesicles stopped growing in size but remained tense and erythematous. In addition, a small erythematous macular rash had appeared on her foot. On day 5 , the patient applied the topical steroid once followed by 2 later applications of an emollient (cocoa butter oil with vitamin E). The tense vesicles on her abdomen and thighs became flaccid and less painful, though new tense vesicles developed on her foot at the site of the previous rash. By day 6 , all of the vesicles had become flaccid and began to scab and became less erythematous and less painful. By day 7, the skin surrounding the scabs became hyperpigmented, and the patient reported the rash was otherwise asymptomatic (Figure 3).

\section{Discussion}

Phytophotodermatitis is a nonimmunologic reaction of phototoxin-contaminated skin with ultraviolet light, such as exposure to direct sunlight. ${ }^{4}$ Two 
Figure 3. Hyperpigmented macules on day 7 .

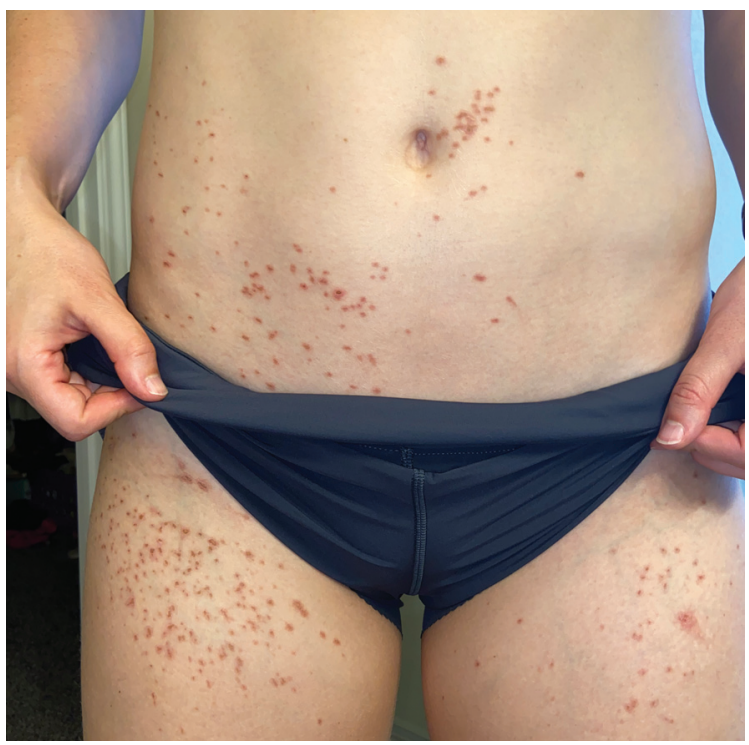

separate biochemical processes are responsible for this cutaneous pathology: one in which ultravioletactivated phototoxins bind to nucleic acids to cause oxygen-independent damage, and another in which these phototoxins directly result in cell membrane damage and edema in an oxygen-dependent reaction. ${ }^{4}$ These 2 phototoxic reactions are responsible for the progressive cell death that presents as this evolving rash. ${ }^{4}$ This patient was exposed to the furocoumarins present in limes through her use of the fruits for a pool party. It is worth noting that limes are the most common cause of phytophotodermatitis. ${ }^{5}$ Following exposure to direct sunlight as she entered the pool, she began to feel the first symptoms of the rash. This is consistent with evidence that wet skin, sweating, and heat all enhance the severity of this phototoxic reaction. ${ }^{4}$

In this case, the sequence of her rash progression indeed follows the clinical triad of this phototoxic reaction. ${ }^{3}$ This patient first complained of erythematous macules following an acute burning sensation in the area. The rash itself was otherwise asymptomatic, until it became more maculopapular and eventually became vesicles. The increase in rubor was accompanied by an increase in symptomatology, as the patient now reported that the rash was tender and burning in sensation. These burning sensations are commonly reported in phytophotodermatitis. ${ }^{5}$ However, it was at this point that the patient's foot began to develop the first phase of this rash with erythematous macules before progressing through the second and third stages normally, and this delayed development is certainly atypical. After a week, the vesicles had ruptured and began to scab over, forming otherwise asymptomatic hyperpigmented macules. This hyperpigmentation is also believed to be mediated by photons: psoralens increase melanocyte mitosis and dendricity, increase tyrosinase activity, induce melanocyte hypertrophy, and effect changes to melanosome size and distribution. ${ }^{5}$ The resulting hyperpigmentation may last for many months before resolving.

The diagnosis of phytophotodermatitis is often made clinically but can be complicated due to its similarity in appearance to many other common cutaneous reactions. ${ }^{6}$ Careful history taking is essential in not only narrowing down the differential diagnosis but also in avoiding unnecessary tests or ineffective treatments. ${ }^{1}$ Solar erythema was ruled out when the patient's seemingly first-degree sunburn began to transform into a seemingly seconddegree sunburn. Contact dermatitis was also considered due to the initial rash appearing along the bikini line, but the patient denied any new clothing or swimwear; the unique transformation of the rash was also an unusual feature. Contact dermatitis secondary to a type IV hypersensitivity reaction to a topical irritant was also excluded once the patient denied any new soaps or sunscreens as well as any possibility of plant exposure besides limes. Another possibility was a chemical burn secondary to cleaning agents in the pool, but this was ruled out once the patient had denied any new cleaning agents being used in the pool and confirmed that she was the only attendee who developed a rash. Polymorphic light eruption was considered unlikely due to any history of sunlight sensitivity or similar rashes in the past as well as the changing nature of the rash being inconsistent with the typical presentation of polymorphic light eruptions. Drug-related photosensitivity was likewise unlikely due to the patient denying any new medications (including nonsteroidal anti-inflammatory drugs).

On clinical diagnosis, management of phytophotodermatitis often depends on severity of symptoms. All patients should be immediately advised to avoid sunlight and photosensitizing agents following the initial reaction. ${ }^{5}$ In general, phytophotodermatitis is typically self-resolving without long-term sequelae and therefore does not require treatment. ${ }^{1}$ There is limited evidence on the efficacy of 
treatment for phytophotodermatitis, ${ }^{6}$ and this may be an area worth future study. However, mild symptoms may see improvement with conservative management via application of a moist dressing. ${ }^{5}$ For moderate symptoms, such as this patient had with increasingly severe burning pain, shortterm corticosteroids or antihistamines may be used for patient comfort. ${ }^{5,6}$ Severe cases may require admission to a burn unit for wound care, such as for patients with phytophotodermatitis involving more than $30 \%$ of total body surface area or cases in which there is severe inflammation and necrosis. ${ }^{5}$ Prevention is the cornerstone of education regarding phytophotodermatitis, as patients should understand that phototoxic agents should be cleansed from the skin before they can be absorbed, meaning that such exposures must be identified and washed with water within 30 to 120 minutes. ${ }^{5}$ Overall, this case may assist clinicians in navigating the diagnostic difficulty of phytophotodermatitis and thereby aid in the recognition and treatment of similar cases.
To see this article online, please go to: http://jabfm.org/content/ 34/2/398.full.

\section{References}

1. Choi JY, Hwang S, Lee SH, et al. Asymptomatic hyperpigmentation without preceding inflammation as a clinical feature of citrus fruits-induced phytophotodermatitis. Ann Dermatol 2018;30:75-8.

2. Melough MM, Cho E, Chun OK. Furocoumarins: a review of biochemical activities, dietary sources and intake, and potential health risks. Food Chem Toxicol 2018;113:99-107.

3. Matthews MR, Vandervelde JC, Caruso DM, et al. Lemons in the Arizona sunshine: the effects of furocoumarins leading to phytophotodermatitis and burn-like injuries. Wounds 2017;29:E118-24.

4. Sarhae KA, Ibrahim A, Fagan SP, et al. Phytophotodermatitis. Eplasty 2013;13:ic57.

5. Mateus JE, Silva CD, Ferreira M, et al. Phytophotodermatitis: still a poorly recognised diagnosis. BMJ Case Rep 2018;2018:bcr-2018-227859.

6. Harshman J, Quan Y, Hsiang D. Phytophotodermatitis: rash with many faces. Can Fam Physician 2017;63:938-40. 